\title{
Safety and effectiveness of kidney transplantation using a donation-after-brain-death donor with acute kidney injury: a retrospective cohort study
}

Kyeong Deok Kim, Kyo Won Lee, Sang Jin Kim, Okjoo Lee, Jieun Kwon, Eun Sung Jeong, Manuel Lim, Jaehun Yang, Jae Berm Park

Division of Transplantation, Department of Surgery, Samsung Medical Center, Seoul, Korea

Background: The use of kidneys from donation-after-brain-death (DBD) donors with acute kidney injury (AKI) is a strategy to expand the donor pool. However, debate continues about the use of donors with AKI. The aim of this study was to evaluate how kidney transplantation (KT) from a donor with AKI affects long-term graft survival in various situations.

Methods: All patients who underwent KT from DBD donors between June 2003 and April 2016 were retrospectively reviewed. The KDIGO (Kidney Disease: Improving Global Outcomes) criteria were used to classify donor AKI.

Results: The cohort included 376 donors (no AKI group, n=117 [31.1\%]; AKI group, n=259 [68.9\%]). The delayed graft function (DGF) rate was significantly higher in the AKI group $(P<0.001)$ and tended to increase with $A K I$ stage $(P<0.001)$. Death-censored graft survival was similar according to the presence of $A K I, A K I$ severity, and the AKI trend $(P=0.929, P=0.077$, and $P=0.658$, respectively). In addition, death-censored graft survival was similar in expanded criteria donor KT ( $P=0.617)$ and the high kidney donor profile index $(\geq 80)$ group $(\mathrm{P}=0.420)$. In the multivariate analysis, patients whose donors had AKI who received using low dose (1.5 $\mathrm{mg} / \mathrm{kg}$ for 3 days) rabbit anti-thymocyte globulin ( $\mathrm{r}-\mathrm{ATG}$ ) as the induction agent had significantly superior death-censored graft survival compared with patients in that group who received basiliximab $(P=0.039)$.

Conclusions: AKI in DBD donors negatively affected the DGF rate. However, it did not affect long-term death-censored graft survival. Low-dose r-ATG can be used for induction immunosuppression in recipients receiving kidneys with AKI because it showed better graft survival than basiliximab.

Corresponding author: Kyo Won Lee

E-mail:kw1980.lee@gmail.com

\footnotetext{
(C) The Korean Society for Transplantation

This is an Open Access article distributed under the terms of the Creative Commons Attribution Non-Commercial License (http://creativecommons.org/licenses/by-nc/4.0/) which permits unrestricted non-commercial use, distribution, and reproduction in any medium, provided the original work is properly cited.
} 